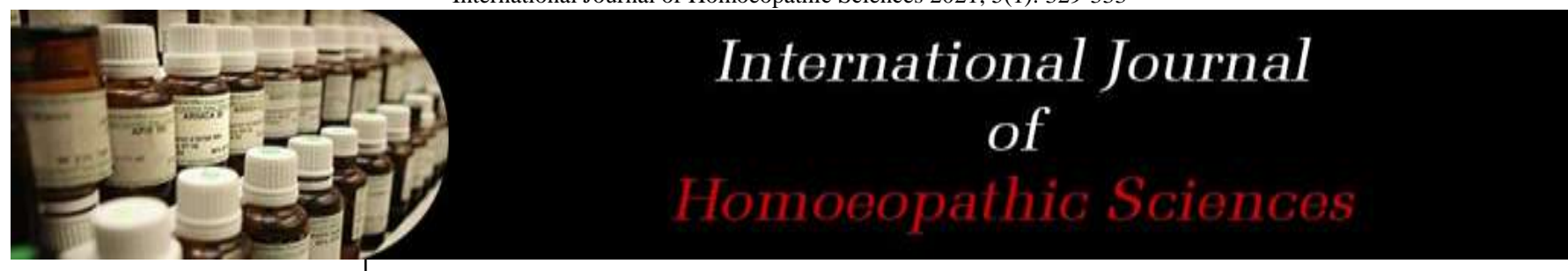

E-ISSN: $2616-4493$

P-ISSN: 2616-4485

www.homoeopathicjournal.com

IJHS 2021; 5(1): 329-333

Received: 11-12-2020

Accepted: 03-01-2021

Dr. Chakravarthy Srigiri

Associate Professor,

Department of Practice of

Medicine, NHRIMH, Kerala,

India

Dr. Jayashree Janagam Assistance Professor,

Department of Psychiatry,

NHRIMH, Kerala, India

Dr. Neelima Mundanattu

Post Graduate Scholars,

NHRIMH, Kerala, India

Dr. Raja Hirams Chebrolu Post Graduate Scholars, NHRIMH, Kerala, India

Corresponding Author: Dr. Chakravarthy Srigiri Associate Professor,

Department of Practice of Medicine, NHRIMH, Kerala, India

\section{A critical review on causation of migraine and its homoeopathic perspective}

\author{
Dr. Chakravarthy Srigiri, Dr. Jayashree Janagam, Dr. Neelima \\ Mundanattu and Dr. Raja Hirams Chebrolu
}

DOI: https://doi.org/10.33545/26164485.2021.v5.i1f.332

\begin{abstract}
Introduction: Migraine a commonest Neurological disorder that often causes one-sided Headache associated with Nausea, vomiting, Irritability, Photophobia, and Phonophobia. According to the International Classification of Headache disorders, there are primarily two types of Migraine basing on the presence or absence of Aura. The disorder is more frequent among Women than Men, and attacks occur as early as in childhood too. Many factors cause migraine such as Environmental factors, Physical factors, Food factors, etc. and most importantly the psychological factors play an important role. Homeopathy is popular for its successful cures in Migraine disorder for the last many decades. This work is an attempt on a critical review of various Causative factors of Migraine and the most indicated Homoeopathic Remedies against them.

Methodology: Common symptoms of the migraine cases were taken from various research articles, scientific literature, and the symptoms were repertorized in RADAR software using Synthesis Repertory and Murphy Repertory. The repertorization was done in 3 methods using all the Common symptoms of Migraine, Second only the causative factors of Migraine, and the third, using only Mind symptoms causing/associated with the attack.

Results: After Repertorizing the common symptoms of Migraine, Phos, Silicea, Bryonia, Lachesis, Ignatia as the top five remedies, the second method showed Phos, Ign, Nux. V, Chin as the top-scoring Remedies and Ignatia, Phos, Nat. $\mathrm{m}$ in the third method of Repertorization.
\end{abstract}

Keywords: Migraine, commonest causative factors, homoeopathic remedies

\section{Introduction}

The name 'Migraine' originally comes from the Greek word Hemicrania, meaning 'half of the head', representing one of the most striking features, that in many cases pain only affects one half of the head. However, pain is felt bilaterally, at the front or the back of the head, more rarely in the face, and rarer still in the body ('Migrainous Corpalgia'). Migraine is experienced at any point by over $20 \%$ of women and over $10 \%$ men ${ }^{[1]}$

The attacks last from 4-72 hours, with a pulsating quality. Which can be mild, moderate, or severe, and is aggravated by routine physical activity or least movement ${ }^{[2]}$. Usually, Migraine is associated with symptoms like vomiting, Nausea, Photophobia, and/ or phonophobia. The attack is due to the activation of Meningeal perivascular pain fibers from intracranial structures and skin and muscles. Migraine Headache depends on (a) activation of the Trigeminovascular pathway by pain signals that originate in peripheral intracranial Nocioceptors, and (b) dysfunction of CNS structures involved in the modulation of neuronal excitability and pain ${ }^{[3,4]}$.

It is observed that females are more vulnerable to migraine due to higher anxiety levels and effect of gender is important when investigating Neuro processing of migraneurs ${ }^{[5]}$

Migraine has two major Sub types: Migraine with an aura which is primarily characterized by the transient focal neurological symptoms that usually precede or sometimes accompany the headache. Some patients also experience a premonitory phase, occurring hours or days before the headache, and a headache resolution phase. Premonitory and resolution symptoms include Hyperactivity, Hypo-activity, and depression, cravings for particular foods, repetitive yawning, fatigue and neck stiffness, and/or pain. Migraine without aura is a clinical syndrome characterized by headache with specific features and associated symptoms such as unilateral location, pulsating quality, moderate or severe pain intensity, aggravation by or causing avoidance of routine physical activity, during headache nausea and/or vomiting or photophobia and phonophobia can occur ${ }^{[6]}$. 


\section{Etiology of migraine}

Many factors tried to explain the causation for migraine but the exact etiology is not known yet some triggering factors are expected to cause them. Several intrinsic or extrinsic factors can trigger migraine attack or migraine activity in the brain.

\section{Triggering factors of migraine ${ }^{[7]}$ \\ Environmental}

- The noise was detected as the second most common trigger factor.

- Those with Chronic headaches may be more sensitive to Environmental lighting, even when they are headache-free.

- Travelling causes Migraine as it triggers Trigeminal and Vestibular Nuclei.

- Sleep disorders were the third most common triggering factor.

\section{Psychological}

Migraine may be considered a psychosomatic disorder, with pain as a somatic expression of psychological distress ${ }^{[8]}$. Stress is assumed to affect primary headache by directly impacting pain production and modulation processes at both the peripheral and central levels ${ }^{[9]}$.

Crying also triggers migraines yet the mechanism is not known. The presence of depression or panic attack disorder is associated with a greater risk of developing a migraine [10].

Generalized anxiety disorder along with changes in mood like tension, irritability, alertness, fatigue, and stress is found to precede the onset of a migraine ${ }^{[11,12]}$.

Changes in mood like tension, irritability, alertness, fatigue and stress are found to precede the onset of migraine ${ }^{[13]}$.

Suppression of anger is seen to have increase pain severity during a migraine and better when in anger expressed ${ }^{[14]}$.

Mollaoglu M, 2012 conducted a study that shows that the most common trigger factors were Emotional stress (79\%), Sleep disturbance (64\%), and Dietary factors (44\%). Sleep and stress were significant trigger factors in patients with Migraine with Aura, whereas environmental factors were important trigger factors in patients with migraine without aura ${ }^{[15]}$.

Migraine is associated with major depressive disorder, bipolar disorder, panic disorder, and social phobia. Migraine in association with various mental health disorders results in poorer health related outcomes compared with migraine or a psychiatric condition alone ${ }^{[16]}$.

\section{Pathophysiology}

Many theories tried to explain the migraine causation but valid theories came out such as

1. Vascular and Neurogenic theories suggest that "all pain is an action violated" and headache is caused by vasodilatation of the cerebral and meningeal arteries [17].

2. Cortical Spreading Depression suggests Neuronal Hyperactivity followed by an area of cortical depression, with activation of the trigeminovascular pain pathway ${ }^{[18]}$.

3. Cortical Hyper-excitability in Migraine theory states that psychophysical studies; visual, auditory, and Somatosensory evoked potentials; Magnetoencephalography; and transcranial magnetic stimulation of the motor cortex evidence a heightened reactivity between migraine attacks. Most studies observed that migraineurs have a reduced threshold for induction of phosphenes when compared with controls, which suggests that a low threshold for activation of cortical Hyperexcitability may characterize migraine [19].

4. Genetic Theory explains that first-degree relatives of patients suffering from migraine with aura had a 4-fold increase of relative risk of migraine while relatives of patients with migraine without aura showed only a 1.9fold risk increase ${ }^{[20]}$.

\section{Clinical features}

It is characterized by multiple phases like Premonitory, aura, Headache, Postdrome, and Interictal ${ }^{[21]}$.

\section{Premonitory phase ${ }^{[22,23]}$}

The majority of migraineurs experience a range of premonitory symptoms as early as 3 days before the headache phase. It involves

- Hypothalamus, causing symptoms like fatigue, depression, irritability, food cravings, and yawning, leading to Brainstem, symptoms like muscle tenderness and neck stiffness,

- Cortex, symptoms like abnormal sensitivity to light, sound, and smell, and

- The limbic system, symptoms like depression and anhedonia in the prodromal phase of a migraine attack.

\section{Aura phase}

The ICHD-3 defines Aura as "one or more transient, fully reversible neurological deficits, of which at least one has to have a unilateral localization, that develop over $5 \mathrm{~min}$ or more and lasts for $60 \mathrm{~min}$. The overlap of the Aura and Headache phases is very common ${ }^{[24,25]}$.

\section{Headache phase}

Characterized as unilateral, pulsating, of moderate to severe intensity, and aggravated by physical activity lasting 4-72 h accompanied by Nausea, Photophobia, and Phonophobia [26].

\section{Post-drome}

Include tiredness, difficulties in concentrating, and neck stiffness ${ }^{[27]}$.

\section{Diagnosis ${ }^{[27]}$}

In most cases, Migraine diagnosis is made by eliciting the Patient's history, Neurological examinations, and appropriate investigations like CT \& MRI. According to the International Classification of Headache Disorders, the following Criteria are followed.

\section{Criteria for migraine without aura}

- At least five attacks fulfilling criteria B-D

- Headache attacks lasting 4-72 hours (untreated or unsuccessfully treated)

- Headache with two of the following four characteristics:

- Unilateral location, Pulsating quality, Severe pain intensity, Aggravation by routine physical activity.

- During headache at least one characteristics among Nausea and/or vomiting/Photophobia and phonophobia 
- Not better accounted for by another ICHD-3 diagnosis.

\section{Migraine with aura}

A. At least two attacks fulfilling criteria B and C

B. Reversible aura like Symptoms, Visual, Sensory Speech and/or language, Motor, Brainstem, Retinal.

C. At least three of the following six characteristics: Two or more Aura symptoms occur in succession with each Aura symptom lasting 5-60 minutes,

\section{Comorbidities with migraine}

In some studies, it was said Hypertension, Stroke, Coronary Heart Disease, Subclinical Vascular Brain lesions, Diabetes Mellitus, Lumbar and Cervical pain, Asthma, Epilepsy, Fibromyalgia, some Psychiatric diseases like Depression, Anxiety, Schizophrenia, Panic disorder, Suicidal ideations, Bipolar disorder, etc., are commonly seen illness ${ }^{[28,29]}$.

\section{Management}

\section{Non-pharmacological treatment}

Many of the non-pharmacologic management of migraine came into practice which includes the application of Cold or Pressure to the Head, Reduction of activity, and sensory input (in a quiet or dark environment and attempts to sleep) with pharmacologic therapies if needed. Other Therapies like Hypnosis, Transcutaneous, Electrical stimulation, Acupuncture, and Occipital or Supraorbital nerve blockade have also been considerable. Other specific treatment includes Relaxation therapy, Cognitive-Behavioral Therapy, Psychotherapy, Hypnosis and Physical measures such as Chiropractic, Osteopathy and Physiotherapy ${ }^{[30]}$.

A case report by Brette. $\mathrm{R}$ et al. has made dietary and lifestyle changes as a recommendation for the treatment of migraine ${ }^{[31]}$.

\section{Methodology}

Common symptoms of the migraine cases were taken from various research articles, scientific literature, and the symptoms were repertorized in RADAR software using Synthesis Repertory and Murphy Repertory. The repertorization was done in 3 methods using all the Common symptoms of Migraine, Second only the causative factors of Migraine, and the third, using only Mind symptoms causing/associated with the attack.

Discussion: In diagnosing a case of Migraine, a lot of patients do complain of some common symptoms of disease which are of less utility to Homoeopath and difficult to select Similimum, but the intensification of the common symptoms are the key guidance in the selection of
Similimum when peculiar characteristic symptoms are unavailable. In this study most of the common symptoms of Migraine cases expressed by the patients were only considered and Repertorized by RADAR Software (Synthesis Repertory) to explore the common remedies indicated against those Rubrics.

The Symptoms are:

1. Headache - One-sided

2. Headache - Nausea with

3. Headache vomiting amelioration

4. Headache aggravated from least movement

5. Headache - Aggravation by Noise

6. Headache - Aggravation by Bright Light

7. Headache - Aggravated by Sun from Exposure to

8. Headache - Aggravated by Travelling/Riding

9. Headache - Aggravated by Strong Odors

10. Headache when hunger/Fasting when.

11. Headache binding head amelioration

12. Headache aggravated before Menstrual periods. Or Headache Menses amel

13. Headache aggravated by Emotions suppressed from

14. Headache Anger when

15. Headache Irritability with

16. Headache from Excitement/Mental exertion

17. Headache from Depression

18. Headache from Loss of sleep

The causative factors and aggravating factors are given under the same headings, hence both were conjointly taken and maximum possible rubrics were taken and repertorized and the chart was shown here under. Some of the rubrics were given intensity gradation 3 as they are the commonest symptoms associated with Migraine and less common symptoms like Menses amel, Tight binding amel, Vertigo. Etc. are given intensity 1 , to see the Indicated remedies. The Repertorization was done in three types:

- Based on All the common symptoms of Migraine including causation, aggravation, amelioration.

- On Causative factors alone.

- On Mental symptoms causing/associated with it.

\section{Results}

1. Basing on all the common symptoms of Migraine (Including causation, aggravation, amelioration): A total of 23 Rubrics were considered by critically extracting the rubrics at the possible extent. The Repertorial result was found to be Phos, Silicea, Bryonia, Lachesis, Ignatia, Bell, Nat.mur, Nux vom, Gels, Calc.

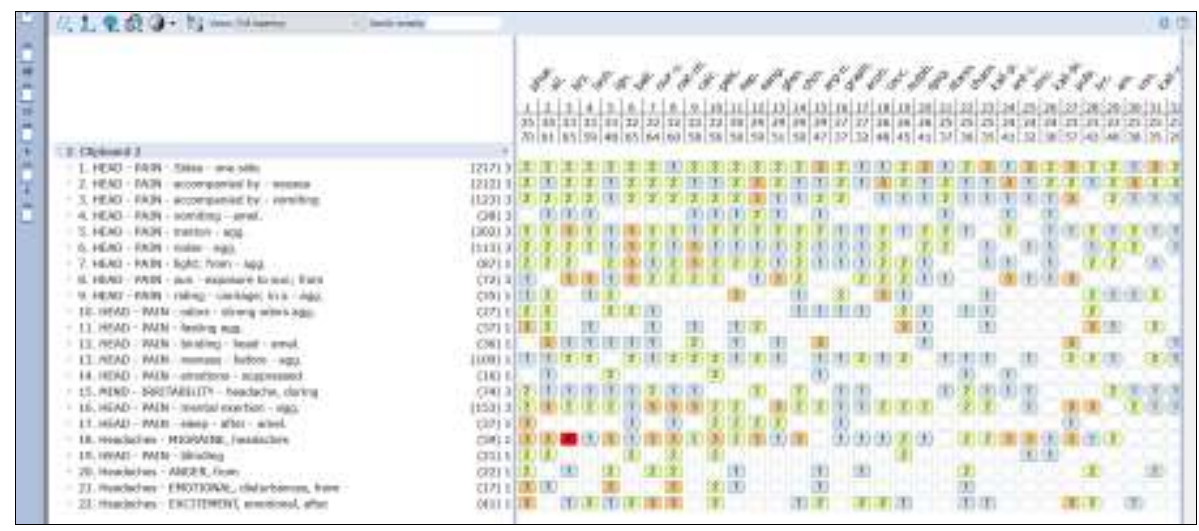

Fig 1: Shows basing on all the common symptoms of Migraine (Including causation, aggravation, amelioration) 
2. Based on only Causative factors: Though many rubrics of causation and aggravation are intermixed with each other we tried to extract rubrics that depict only causative factors expressed by most of the patients. In this the Repertorial result was found to be Phos-15/8; Nux.v-14/8, Ign-13/8; Chin-12/7; Cocc-12/7; Acon - 9/7; Lyco-13/6; Nat.m-11/6.

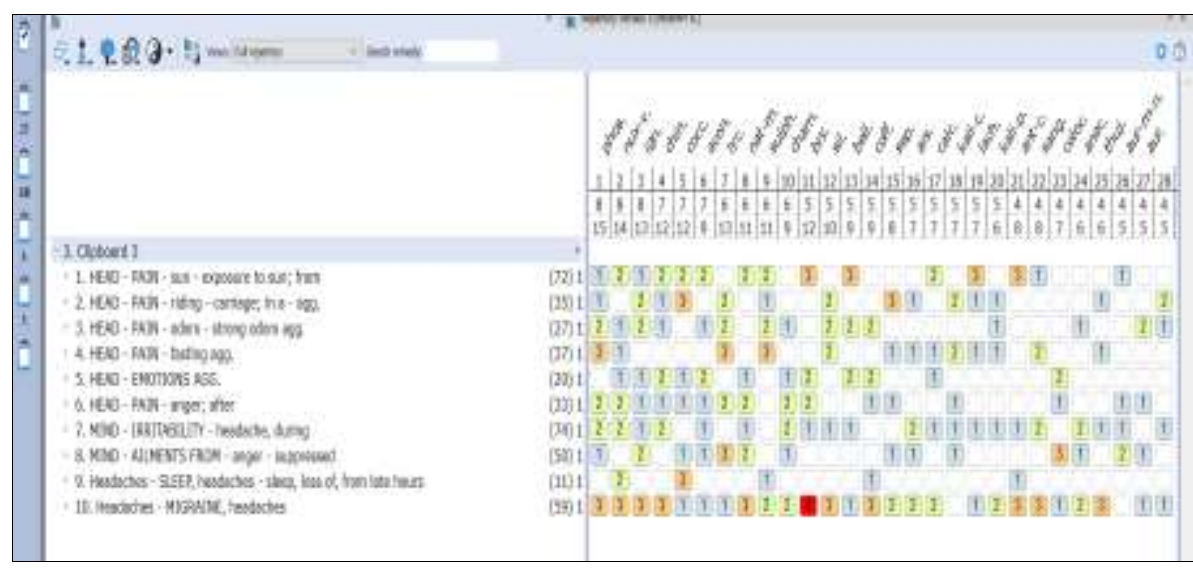

Fig 2: Shows based on only Causative factors

3. Based on only Mind Symptoms as causative/triggering factors: Though many factors cause Migraine, Psychiatric conditions play a major role in causing Headaches especially Migraine. The Symptoms like Headache anger after, Headache emotions suppressed after, Headache
Mental exertion after, Headache Irritability with, etc. are taken and Repertorized to see the probable remedies. The Remedies were Ign- 13/6; Phos-11/5; Nat.m-10/5; Cham9/5, Coff - 9/5, Aur.m-8/5, Puls - 11/4, Bry - 10/4.

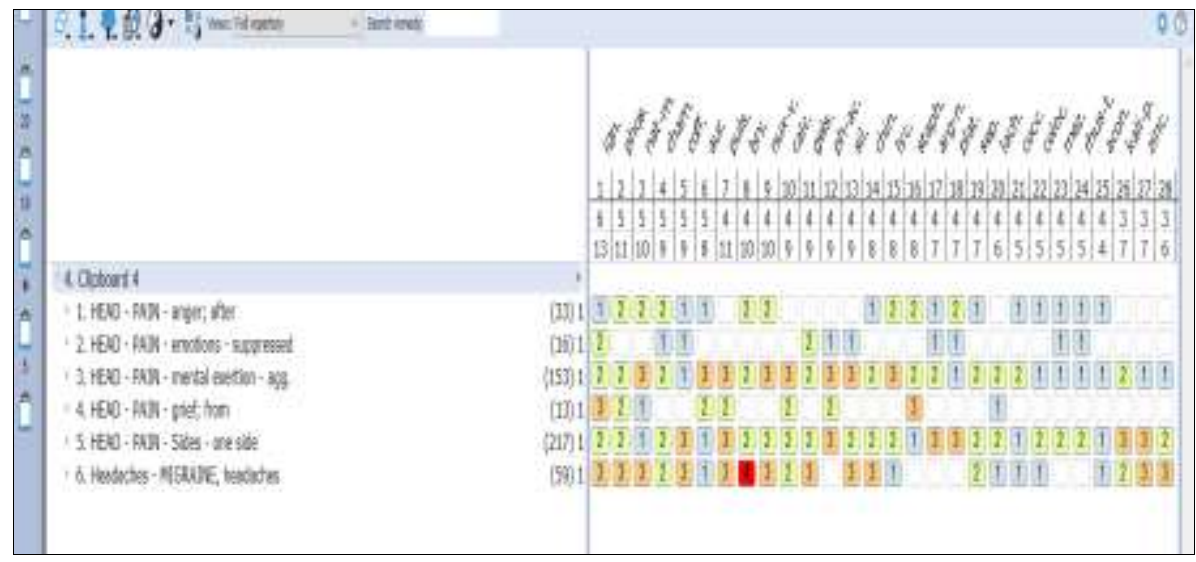

Fig 3: Shows based on only Mind Symptoms as causative/triggering factors

\section{Conclusion}

Migraine the one among the commonest cause of Headaches is prevalent more in women than in men and often in children too. The attack lasts from 4-72 hours, reaching its peak in the duration of $24 \mathrm{hrs}$ of onset. Most of the cases present with or without Aura usually accompanying features like Nausea, Vomiting, etc. Homeopathic Remedies with their action on symptom similarity have a significant curative effect on all Chronic Headaches. Though Homoeopathic Prescription is effective when based on Peculiar, rare striking keynote symptoms, the Common symptoms when intensified can be taken as Symptoms of importance in the hierarchy and can be prescribed as per the need. These symptoms of Migraine were collected, Repertorized in RADAR Software using Synthesis Repertory and few Rubrics from Murphy Repertory. The Repertorization was done in 3 ways using the Common symptoms of Migraine, Only causative factors, and the Third using only the Mental symptoms. After Repertorizing, the first method showed Phos, Silicea, Bryonia, Lachesis, Ignatia as the top five remedies, the second method showed
Phos, Ign, Nux. v, Chin as the top-scoring Remedies and Ignatia, Phos, Nat.m in the third Repertorization Chart. More Polychrest Remedies were also reflected when considered the remaining list.

\section{Note}

This work is only identification of outcome of Homoeopathic remedies for the common symptoms of Migraine sufferers, and only a suggestion of probable remedies that aid in quick prescriptions for the physicians but Individualizing symptoms aiding in selection of constitutional are always of Prime Importance for complete cure, if it can be elicited out from the patients.

\section{References}

1. Mark Weatherall. The diagnosis and treatment of chronic migraine. Therapeutic Advances in Chronic Disease 2015;6:115-123.

2. Poojitha Mamindla et al. A Review on Migraine, Acta Scientific Pharmaceutical Sciences 2019;3(1):1.

3. Fransesca Pulleda. An update on Migraine - Current 
understanding and future directions, Journal of Neurology; Published online 2017.

4. Peter $\mathbf{J}$ et al. Migraine and Other Primary Headache Disorders, Harrison's Principles Of Internal Medicine P2586.

5. Yunuiangguo et al. Female vs Male Migraine, an event related potential study of visual neurocognitive processing. The Journal of Headache and pain, Article number: 38 2019;20.

6. Headache Classification Committee of the International Headache Society. The International Classification of Headache Disorders, 3rd edition. Cephalagia 2013;33(9):629-808.

7. Rama K et al. Study of Triggers of Migraine in India, Pain Medicine. American Academy of Pain Medicine 2010;11:44-47.

8. Lanzi $\mathrm{G}$ et al. Psychological aspects of migraine in childhood. Cephalalgia 1983;3(1);218-20.

9. Spierings EL. Precipitating and aggravating factors of migraine versus tension-type headache. Headache 2001;41:554.

10. Seng EK et al. Psychological factors associated with chronic migraine and severe migraine-related disability: an observational study in a tertiary headache center. The Journal of Head and Face Pain 2017;57(4):593604.

11. Kokane S et al. Anxiety, Depression and Lifestyle Patterns among Adolescents from India with Primary Headache 4456.

12. Bhatia MS, Gupta R. Migraine: clinical pattern and psychiatric comorbidity. Industrial psychiatry journal 2012;21(1):18.

13. Lipton RB. SR. Reduction in perceived stress as a migraine trigger: testing the "let-down headache" Hypothesis. Neurology 2014;82(16):1395-401.

14. Nicholson RA et al. Psychological Risk Factors in Headache: CME. Headache: The Journal of Head and Face Pain 2007;47(3):413-26.

15. Mallaoglu M. Trigger factors in migraine patients. J Health Psychol 2012;18(7):984-94.

16. Jette N. Comorbidity of migraine and psychiatric disorders. A National population-based study. Headache: The Journal of Head and Face Pain 2008;48(4):501-16.

17. Gasparini CF et al. Studies on the pathophysiology and genetic basis of migraine. Curr Genomics 2013;14(5):300-15.

18. Gooriah R et al. Evidence-based treatments for adults with migraine. Pain. Res. Treat. Published online 2015.

19. Rogawski Miachel A. Common Pathophysiologic Mechanisms in Migraine and Epilepsy. Arch. Neurol 2008;65(6):709-14.8.

20. Russell MB et al. Increased familial risk and evidence of genetic factor in migraine 1995;311(7004):541-544.

21. David W et al. A Phase-by-Phase Review of Migraine Pathophysiology. American Headache Society, Published by Wiley Periodicals 2018.

22. Werner Becker J. The Premonitory phase of Migraine and Migraine Management 2013;33(13):1117-21.

23. Rami Burstein et al. Migraine: Multiple Processes, Complex Pathophysiology. The Journal of Neuroscience 2015;35:6619-6629.

24. Rasmussen BK. Migraine with aura and migraine without aura: an epidemiological study. Cephalalgia
1992;12:221-228.

25. Hansen JM et al. Migraine headache is present in the aura phase: A prospective study. Neurology 2012;79:2044-2049.

26. Olesen $\mathbf{J}$ et al. New appendix criteria open for a broader concept of chronic migraine. Cephalalgia 2006;26:742746.

27. Cephalalgia. International Classification of Headache disorders, $3^{\text {rd }}$ Edition 2018;38(1):1-211.

28. Rigmor Jensen et al. Epidemiology and comorbidity of headache. Lancet Neurology 2008;7:354-361.

29. Shuu-Jiun Wang et al. Comorbidities of Migraine. Front Neurology 2010;1:16.

30. William EM et al. Guidelines for the nonpharmacologic management of migraine in clinical practice. Can Med Assoc 1998;159(1):47-54.

31. Brett $\mathrm{R}$ et al. Dietary and Lifestyle Changes in the Treatment of a 23-Year-Old Female Patient With Migraine. National University of Health Sciences 2015;14(3):205-11. 